\title{
Magnetic Resonance Microscopy Contribution to Interpret High-Resolution Magic Angle Spinning Metabolomic Data of Human Tumor Tissue
}

\author{
M. Carmen Martínez-Bisbal,, ${ }^{1,2}$ Vicent Esteve, ${ }^{1,2}$ Beatriz Martínez-Granados, ${ }^{2}$ \\ and Bernardo Celda ${ }^{1,2}$ \\ ${ }^{1}$ Networking Research Center on Bioengineering, Biomaterials and Nanomedicine (CIBER-BBN), ISC-III, C/Doctor Moliner 50,
46100 Burjassot, Valencia, Spain
${ }^{2}$ Department of Physical Chemistry, University of Valencia, C/Doctor Moliner 50, 46100 Burjassot, Valencia, Spain
}

Correspondence should be addressed to M. Carmen Martínez-Bisbal, carmen.martinez-bisbal@uv.es

Received 20 April 2010; Revised 16 July 2010; Accepted 3 August 2010

Academic Editor: Mika Ala-Korpela

Copyright ( $) 2011$ M. Carmen Martínez-Bisbal et al. This is an open access article distributed under the Creative Commons Attribution License, which permits unrestricted use, distribution, and reproduction in any medium, provided the original work is properly cited.

HRMAS NMR is considered a valuable technique to obtain detailed metabolic profile of unprocessed tissues. To properly interpret the HRMAS metabolomic results, detailed information of the actual state of the sample inside the rotor is needed. MRM (Magnetic Resonance Microscopy) was applied for obtaining structural and spatially localized metabolic information of the samples inside the HRMAS rotors. The tissue was observed stuck to the rotor wall under the effect of HRMAS spinning. MRM spectroscopy showed a transference of metabolites from the tissue to the medium. The sample shape and the metabolite transfer after HRMAS indicated that tissue had undergone alterations and it can not be strictly considered as intact. This must be considered when HRMAS is used for metabolic tissue characterization, and it is expected to be highly dependent on the manipulation of the sample. The localized spectroscopic information of MRM reveals the biochemical compartmentalization on tissue samples hidden in the HRMAS spectrum.

\section{Introduction}

NMR is a very valuable tool for the study of healthy or diseased brain given the possible translation of that knowledge to the clinical practice. Brain tumors are among the most studied pathologies by NMR in its different modalities. In vivo NMR spectroscopy (MRS) can support tumor presurgical diagnosis (tumor identification, border delineation and infiltration detection) as well as treatment monitoring [1-5]. In in vivo MRS the spatial and spectral resolution are limited. With the purpose to obtain a better metabolite identification and quantification, high-resolution NMR has been applied to the tumor tissue ex vivo. These studies were initially performed in solution, and required a previous chemical extraction with Perchloric acid or organic solvents [6, 7]. High-Resolution Magic Angle Spinning (HRMAS) NMR has become in recent years a valuable technique to obtain detailed metabolic profiles of brain tumors [8-13]. This technique requires low tissue amount (ca. $20 \mathrm{mg}$ ) and minimum sample handling compared to chemical extracts. Probably because this limited processing of the sample [9-11], HRMAS is considered a metabolomic technique that provides "intact" tissue spectra. Nevertheless, some researchers have shown some reluctance to assume the integrity of the tissue due to the sample handling and measurement conditions. An issue of interest is the postmortem interval for tissue obtained from autopsies, or in the case of pieces from resection, the time that the sample remains in the surgery room before being preserved. The results so far obtained show that this time period between tissue death and tissue preservation causes changes in the tissue metabolite concentration $[8,14]$ as might be expected. Knowing this effect, the temperature to run the experiments needs to be low to preserve as much as 
possible the biochemical profile. In fact, comparing the biochemical profiles of experiments at $20^{\circ} \mathrm{C}$ and $2^{\circ} \mathrm{C}$, there were 4 -fold reduction in the content of some metabolites at $20^{\circ} \mathrm{C}$ compared to $2^{\circ} \mathrm{C}$ [8], which indicates the tissue degradation at $20^{\circ} \mathrm{C}$. However, it has also been found that for tissue maintained at $4^{\circ} \mathrm{C}$ and spinning at $5 \mathrm{kHz}$ there were more significant changes in the metabolite concentrations over 4 hours of measurement than the changes due to the delayed initial freezing [14]. The cellular damage by high spinning speed of HRMAS seems to be promoting some release of metabolites that previously were NMR-invisible [14]. Finally, the effect of freezing-thawing the tissue samples has been of interest for some researchers $[15,16]$. Freezing and thawing of any tissue is likely to cause unpredictable amounts of cell damage and lysis [16]. In some tissues there have been reported changes in the molecular composition of the samples frozen, compared to samples studied without previous freezing. According to these researchers, tissue samples that are subjected to freezing will undergo some physical disruption of cellular compartmentalization and changes in molecular composition [15]. Moreover, the tissues thawed after freezing show that a great amount of metabolites (40\%-50\% for human prostate and $20 \%$ for rat brain) leak into the storage buffer at the time that thawing is completed, and the amount of a given metabolite observed in the medium varies from one biopsy to another and is not proportional to the amount originally present in the tissue [16].

Therefore, a proper biochemical interpretation of the HRMAS results would require detailed and localized molecular information on the tissue that can be correlated with the histopathological analysis and other molecular characterization techniques. It would be advisable as well to know the actual state of the tissue inside the rotor and a direct visualization could be of help, providing, as additional information, the overall shape of the sample. This study shows the potential role of NMR microscopy, MRM (Magnetic Resonance Microscopy), in obtaining structural and spatially localized metabolic information of the tissue samples inside the HRMAS rotors and to study the real integrity of the tissue in HRMAS. With this objective MRM imaging, MRM spectroscopy, and HRMAS has been applied to the study of human Glioblastoma Multiforme (GBM) biopsies.

Gliomas are the most common primary brain tumors. GBM is the highest glioma grade and the most common malignant central nervous system tumor in adults $[17,18]$.

\section{Materials and Methods}

This study was reviewed and approved by the ethics committee of the Hospital de la Ribera, Alzira, Spain. Informed consent was obtained from all patients as a part of eTUMOUR Project (FP6-2002-LSH 503094). Eight GBM samples (A$\mathrm{H}$ ) were studied by MRM and HRMAS inside $4 \mathrm{~mm} / 50 \mu \mathrm{L}$ $\mathrm{ZrO}_{2}$ rotors. Sample preparation was done following already published procedures [9]. The amount of tumoral tissue ranged from 9.8 to $27.3 \mathrm{mg}$ (see Table 1). This quantity was split from the whole tumoral mass submerged in liquid nitrogen. Samples were introduced in the rotor without flushing, thus avoiding the leakage of metabolites reported in the washing of tissues [16]. The volume of the rotor was fitted with cool $\mathrm{D}_{2} \mathrm{O}$. Then, the rotor was transferred into the NMR probe precooled to $6^{\circ} \mathrm{C}$. In both probes, MRM and HRMAS, the real temperature was internally assessed using a $100 \% \mathrm{MeOH}$ sample in a $4 \mathrm{~mm}$ rotor in the same conditions than the samples.

Several settings were tested: MRM and HRMAS experiments were performed on two tissue samples extracted from the same tumor (samples A-E), MRM before HRMAS (sample F), and MRM after HRMAS (samples G and H). Moreover, in sample $G$ the solution part was separated from the tissue at the end of the HRMAS and MRM studies and introduced in a $4 \mathrm{~mm} / 12 \mu \mathrm{L}$ rotor to acquire an additional HRMAS CPMG spectrum. Details of the experiments performed on each sample are given in Table 1.

MRM studies were conducted in a $14 \mathrm{~T}$ magnet (Bruker Avance DRX 600 spectrometer operating at a ${ }^{1} \mathrm{H}$ frequency of $600.13 \mathrm{MHz}$ ) connected to a microimage console with a gradient system of $300 \mathrm{G} / \mathrm{cm}$ at $60 \mathrm{~A}$ current (Bruker Biospin). The instrument was equipped with a standard bore Micro 5 imaging probehead with an $\mathrm{rf}$ insert of $5 \mathrm{~mm}$. A Bruker Cooling Unit Extreme controlled the temperature during the acquisition. HRMAS experiments were conducted in an $11.7 \mathrm{~T}$ magnet (Bruker Avance DRX 500 spectrometer operating at a ${ }^{1} \mathrm{H}$ frequency of $500.13 \mathrm{MHz}$ ). The instrument was equipped with a $4 \mathrm{~mm}$ triple resonance ${ }^{1} \mathrm{H} /{ }^{31} \mathrm{P} /{ }^{13} \mathrm{C}$ HRMAS probe. A Bruker cooling unit was used to control the temperature by cooling down the bearing air flowing into the probe. Sample temperature was $6^{\circ} \mathrm{C}$ for MRM and HRMAS experiments.

In the MRM study of each sample, a global shimming was performed first, including the whole content of the rotor. The tissue pieces were then localized inside the rotors using imaging multislice sequences RARE (Rapid Acquisition with Relaxation Enhancement) with TR/TE 2000/80 ms and MDEFT (Modified Driven-Equilibrium Fourier Transform) with TR/TE 5000/4.5 ms adapted to MRM. In these RARE and MDEFT images, the FOV was $10 \mathrm{~mm}$ and the slice thickness was $500 \mu \mathrm{m}$. The matrix dimensions were $256 \times$ 256 elements, which gave an in-plane spatial resolution of 39 $\times 39 \mu \mathrm{m}$. MRM-localized spectroscopy was performed with PRESS (Point resolved spectroscopy) single voxel (TR/TE $2100 / 12 \mathrm{~ms}$ ). Before acquiring each single voxel spectrum, a specific shimming was performed, shimming only the volume determined by each single voxel and optimizing the 1st-order shims. This shimming procedure was repeated in each location, tissue and solution, for each sample. VAPOR (Variable pulse power and optimized relaxation delays) was used for water suppression. The spectral width was $10 \mathrm{ppm} / 6000 \mathrm{~Hz}$ and the number of points was $4 \mathrm{k}$. Depending on the volume selected, the number of repetitions was from 512 to 4096. Single-voxel PRESS spectroscopy was performed in the tissue, and the solution parts in samples AG. Single-voxel in sample $\mathrm{H}$ was acquired only in the solution part, since the tissue piece was a thin slice stuck to the rotor wall. 
TABLE 1: MRM and HRMAS experiments for each tumor and sample.

\begin{tabular}{|c|c|c|c|c|c|c|}
\hline GBM tumor & $\begin{array}{c}\text { Sample } \\
\text { ID }\end{array}$ & $\begin{array}{l}\text { Weight (mg) } \\
\text { MRM } \\
\end{array}$ & $\begin{array}{l}\text { Weight }(\mathrm{mg}) \\
\text { HRMAS }\end{array}$ & Medium & $\begin{array}{l}\text { MRM single-voxel } \\
\text { dimensions (mm) }\end{array}$ & HRMAS performance \\
\hline 1 & A & 20.6 & 21.5 & \multirow{3}{*}{$\mathrm{D}_{2} \mathrm{O}$} & $1 \times 1 \times 1$ & \multirow{5}{*}{$\begin{array}{l}\text { Parallel: other piece } \\
\text { from the same tumors }\end{array}$} \\
\hline 2 & B & 16.7 & 17.3 & & $1 \times 1 \times 1$ & \\
\hline 3 & $\mathrm{C}$ & 15.2 & 20.0 & & $1 \times 1 \times 1$ & \\
\hline 4 & $\mathrm{D}$ & 9.8 & 22.7 & & $1.5 \times 1.5 \times 1.5$ & \\
\hline 5 & $\mathrm{E}$ & 13.0 & 22.1 & & $1 \times 1.8 \times 1.8$ & \\
\hline 5 & $\mathrm{~F}$ & \multicolumn{2}{|c|}{24.7} & $\begin{array}{l}\text { PBS in } \\
\mathrm{D}_{2} \mathrm{O}\end{array}$ & $1.2 \times 1.2 \times 1.2$ & after MRM \\
\hline 6 & G & \multirow{2}{*}{\multicolumn{2}{|c|}{13.9}} & & $1 \times 1 \times 1$ & \multirow{2}{*}{ before MRM } \\
\hline 4 & $\mathrm{H}$ & & & & $1.5 \times 1.5 \times 1.5$ & \\
\hline
\end{tabular}

In HRMAS experiments samples were spun at $4000 \mathrm{~Hz}$. The shimming was optimized checking the quality of the signals and the resolution of the alanine doublet, usually present in this kind of samples. HRMAS proton CPMG spectra (Carr-Purcell-Meilboom-Gill) was performed with a 2D CPMG sequence with presaturation in the water signal. 2D CPMG spectra were acquired with a spectral width of $12 \mathrm{ppm} / 6000 \mathrm{~Hz}$ and the number of points in F2 was $16 \mathrm{k}$. The delay was fixed to $2 \mathrm{~ms}$ and the scan with 4 loops gave and effective TE of $16 \mathrm{~ms}$. This was the nearest TE for comparison with MRM PRESS single-voxel TE $12 \mathrm{~ms}$.

\section{Results and Discussion}

MRM images of the samples without previous HRMAS rotation (A-F) showed the tissue suspended in the solution and without any relevant deformation of the piece. Examples of these images (samples D and E) are shown in Figure 1. On the contrary, MRM images in samples $\mathrm{G}$ and $\mathrm{H}$ clearly showed the disposition of the tissue close to the rotor wall, due to the effect of high spinning speed on the previous HRMAS study (see MRM axial images in Figure 1). In the samples $G$ and $H$, in which a previous HRMAS study was acquired, the solution part that remained in the central axis of the rotor ( $\mathrm{PBS}$ in $\mathrm{D}_{2} \mathrm{O}$ ) contained a considerable amount of metabolites. This fact is clearly seen in the MRM singlevoxel spectrum of sample G (Figure 2(g), middle spectrum); the main resonances were assigned to lactate, alanine, lipids, creatine, choline, and phosphocholine, glycine and mannitol (peaks are assigned in Figure 2(g), top spectrum) [9]. The single-voxel spectrum in the tissue of sample G (Figure 2(g), bottom spectrum) showed lower resolution than in solution (Figure 2(g) middle spectrum), but still could be observed lipids, creatine, choline, and mannitol resonances. In this later single voxel, the tissue location close to the rotor wall seriously precluded an adequate field homogeneity.

The HRMAS study of the separated solution part from sample G (after the HRMAS and MRM study) confirmed the metabolical content of the solution using the same technique (Figure 3(d)). The resulting metabolic profile of the solution was similar to the previous HRMAS CPMG spectrum in the sample and to the MRM single-voxel spectrum in the solution. The observation of this solution with a magnifying glass (50x) assessed the absence of any tissue or cell in this solution.

The samples either with pure $\mathrm{D}_{2} \mathrm{O}(\mathrm{A}-\mathrm{C})$ or PBS in $\mathrm{D}_{2} \mathrm{O}$ (D-F), but without previous rotation, also showed transference of metabolites from the tissue to the medium (Figure 2, middle spectra). A similar leakage of metabolites had been reported in previously frozen samples [16]. The metabolites appeared in the buffer solution ( $\mathrm{PBS}$ in $\mathrm{D}_{2} \mathrm{O}$ ) immediately after thawing, and the amount of a given metabolite observed in the medium varied from one biopsy to another and was not proportional to the amount originally present in the tissue [16]. Figure 2 gathers the MRM singlevoxel spectra from the tissue and the solution for all the samples in this study. In all the cases the single-voxel spectra in the solution showed the presence of metabolites.

There were substantial differences between the MRM single-voxel spectra in solution and tissue parts. The most striking differences were observed on the lipids, which almost preponderantly appeared in the tissue single-voxel spectra (it is very evident in samples A and C, see Figures 2(a) and 2(c), bottom and middle spectra). In fact, the lipids are commonly observed on GBM in vivo and ex vivo spectra and are usually related to their aggressiveness. Moreover, quantitative differences could be observed between the solution and the tissue parts in other metabolites: in sample B the choline derivatives proportion in the tissue and the solution was different, and in sample $\mathrm{E}$ the alanine was undetectable in the tissue but it was present in the solution part.

Noteworthy, in the samples here studied the global metabolic profiles observed either by MRM or by HRMAS showed some significant differences, which is in agreement with the heterogeneous nature of GBM tumor (areas of actively growing tumor, necrosis, border infiltration,...) [17]. The metabolite transfer from the tissue to the solution was also different, which agrees with previously published studies on tissues [16], despite being preserved and studied by the same procedures. An example of these differences can be observed in the samples $\mathrm{C}$ and D (Figures 2(c) and 2(d)). According to this, the percentage of the contribution of the solution and tissue spectra to the whole HRMAS spectrum might not be the same for each sample. In the sample C the lipid content is high (as seen in HRMAS and MRM tissue spectra) and a large part of these molecules remains 


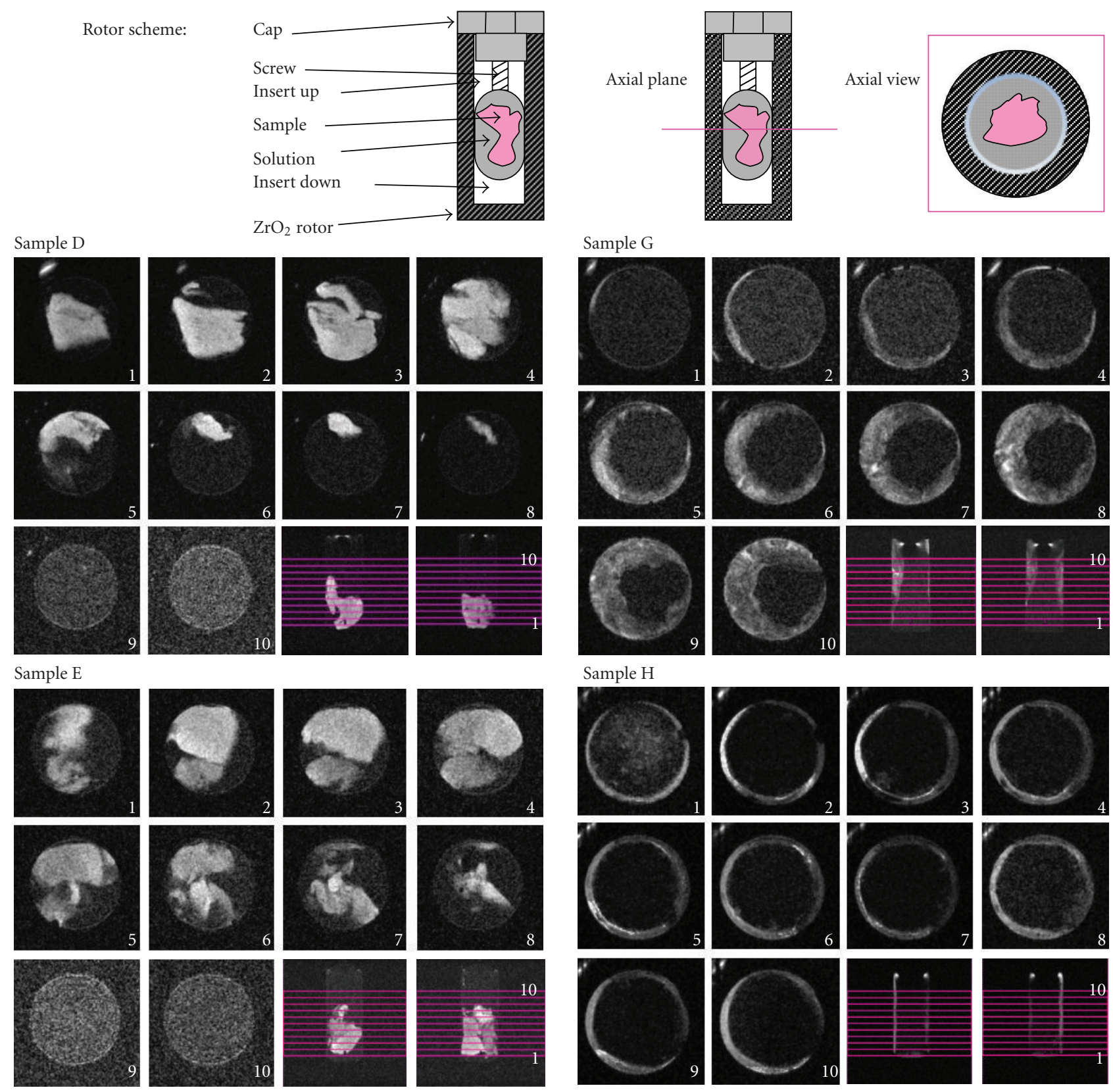

FIGURE 1: The upper part shows the scheme of a rotor containing the sample and the solution and the axial MRM images of four samples. The scheme of the HRMAS rotor shows the different parts with the sample and the solution in the inner cavity. The position of the axial plane is drawn with a magenta line and the axial view of the rotor on the right (upper part) shows the axial section, which is perpendicular to the longitudinal axis of the rotor. Below, the set of MRM images shows 10 RARE axial slices and the central sagital and coronal planes for each of the samples showing the shape of tissue pieces inside the rotor for samples D, E, G, and H. The position of the 10 axial images is detailed by the 10 magenta horizontal lines in the sagital and coronal planes in each sample. Axial images are zoomed to achieve the optimal view of the sample. In these MRM RARE images the solution, the rotor, and the air outside the rotor are darker than the tissue.

in the tissue whereas a small amount of low molecular weight metabolites are transferred to the liquid environment (Figure 2(c)). In this case, the main contribution to the final global spectrum will came from the tissue. On the contrary, sample D shows a low amount of lipids and hence, an important part of low molecular weight metabolites is observed in the solution, and the linear combination of solution and tissue spectra will be different to the one for the sample C.

In the case of sample $\mathrm{H}$, that underwent HRMAS previous to the MRM study, the effect of metabolite transference is such that even some lipids signals appear clearly in the MRM spectrum in the solution part (Figure 2(h), bottom spectrum). As it has been demonstrated in previous studies 


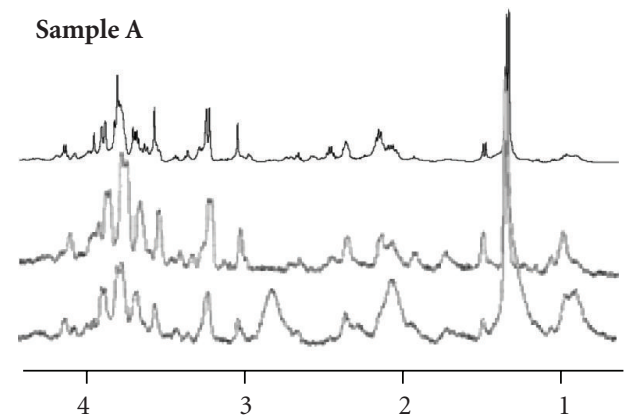

(a)

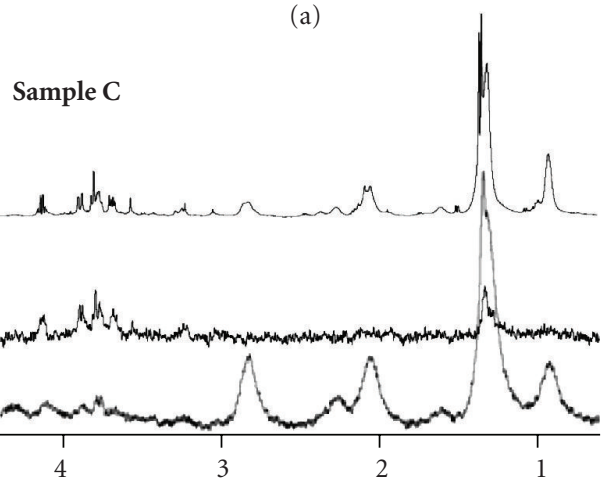

(c)

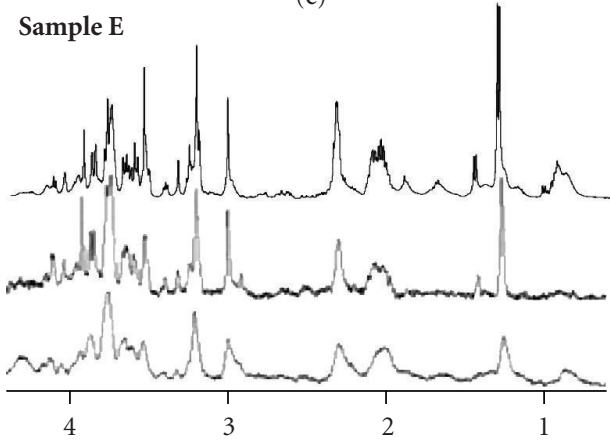

(e)

Sample G

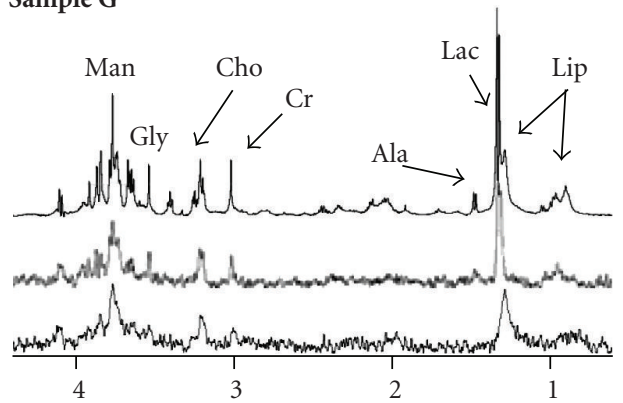

(g)

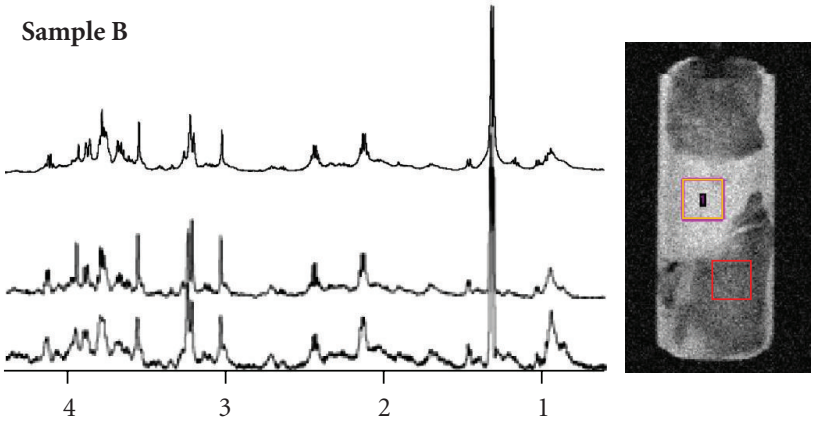

(b)
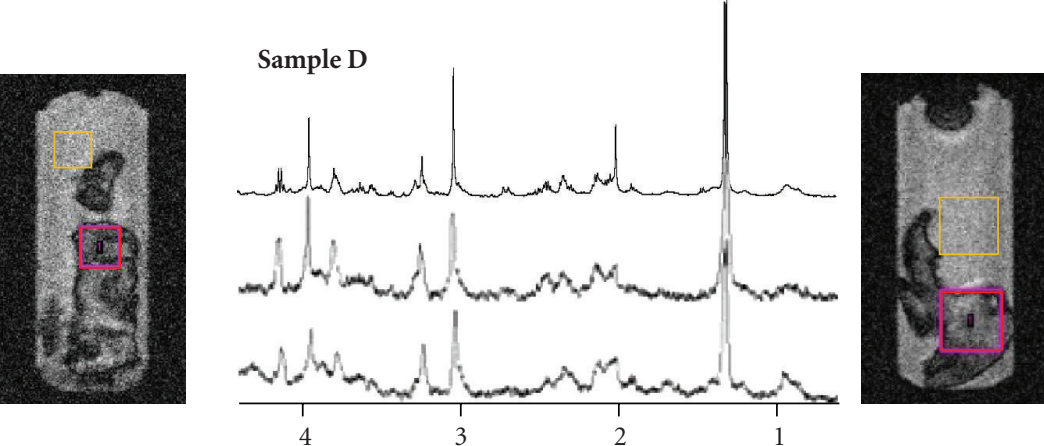

(d)
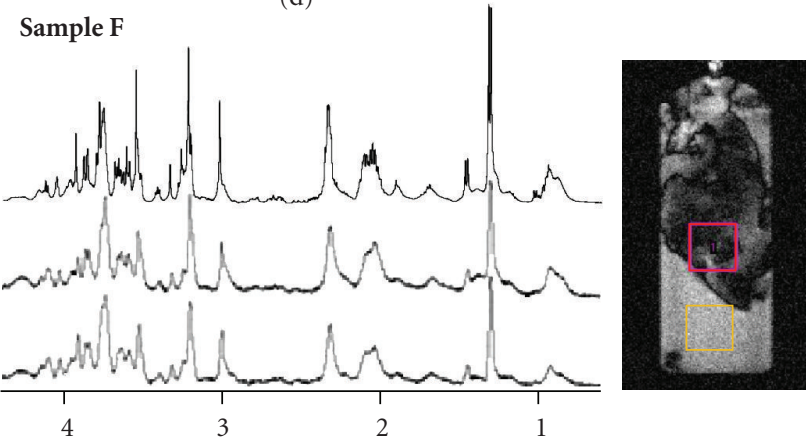

(f)

Sample H
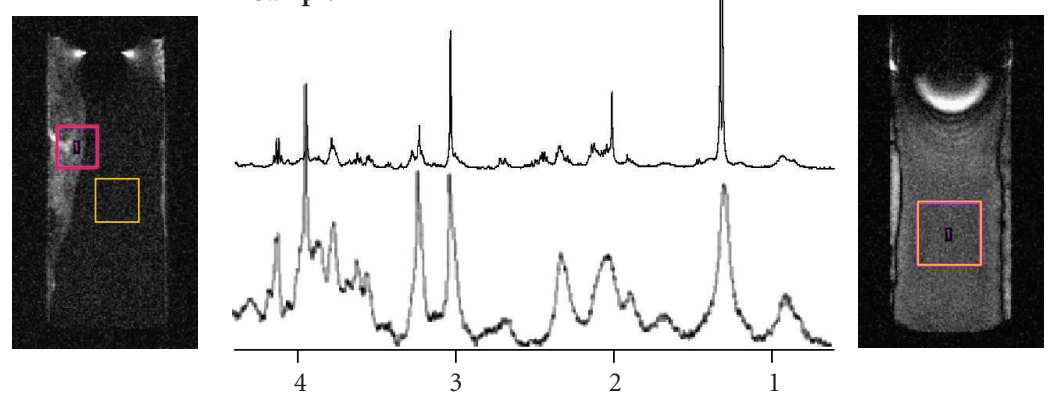

(h)

FIgURE 2: HRMAS CPMG (top) and MRM spectroscopy PRESS single-voxel spectra from solution (middle) and tissue (bottom) in each of the samples (A-H). MRM single-voxel location in tissue (red box) and solution (yellow box) is illustrated in the MRM images. Samples G and $\mathrm{H}$ were the only samples which underwent HRMAS experiments before MRM. Sample F underwent HRMAS study after MRM. The samples A-E underwent parallel study by HRMAS in other piece from the same biopsies. The resonances assigned in the CPMG HRMAS spectrum of sample G are: mannitol (Man), glycine (Gly), choline and phosphocholine (Cho), creatine (Cr), alanine (Ala), lactate (Lac), and lipids (Lip). For each sample, the MRM images shown are those that better illustrate the tissue shape and position inside the rotor. The MRM images shown for samples A and G belong to RARE sequences and the MRM images for samples B-F and H belong to MDEFT images. Moreover, the contrast in these MRM image was balanced to enhance the differences between the tissue and the solution part. 

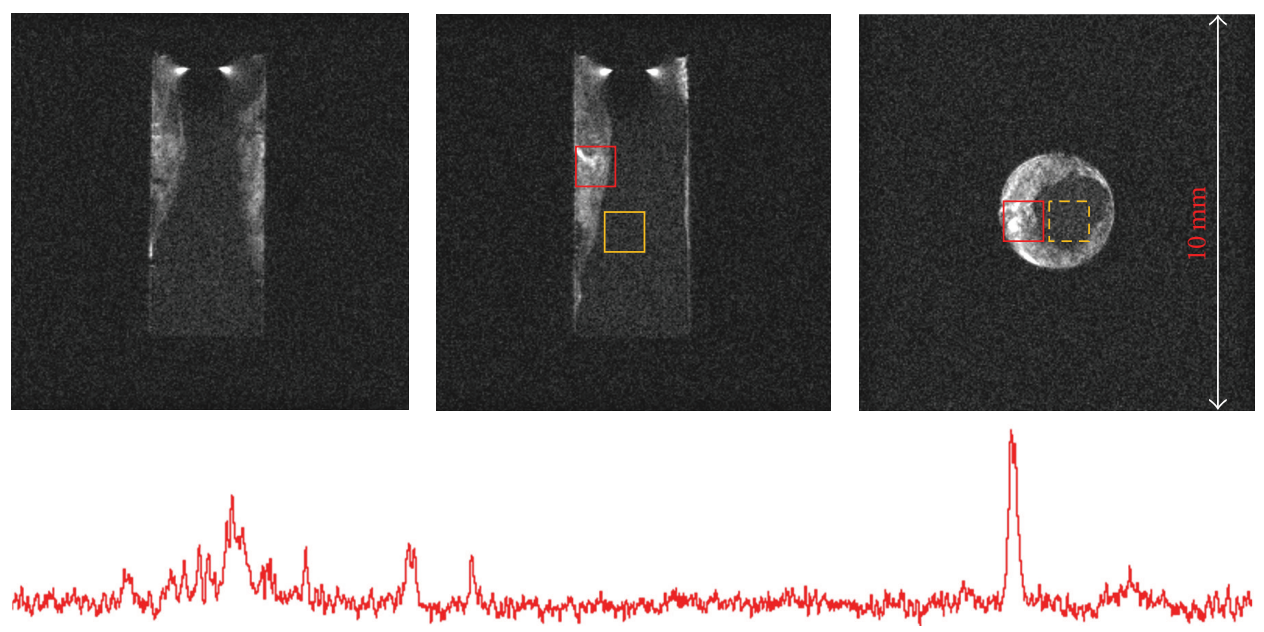

(a)

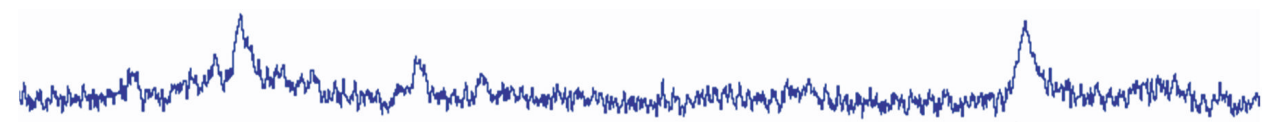

(b)

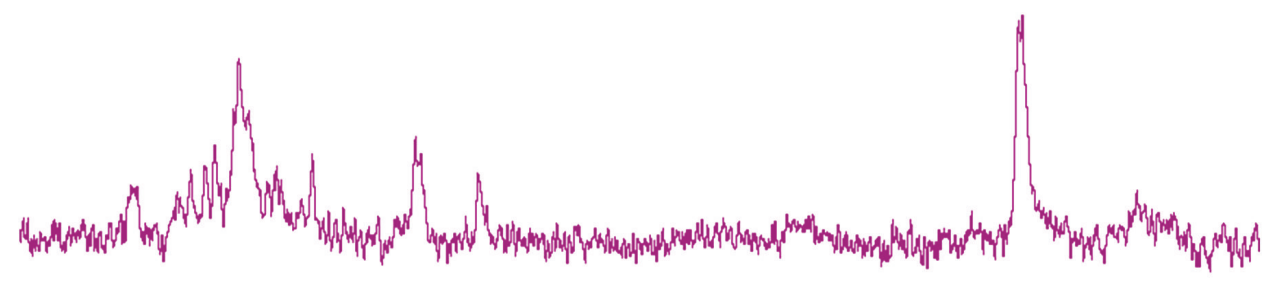

(c)

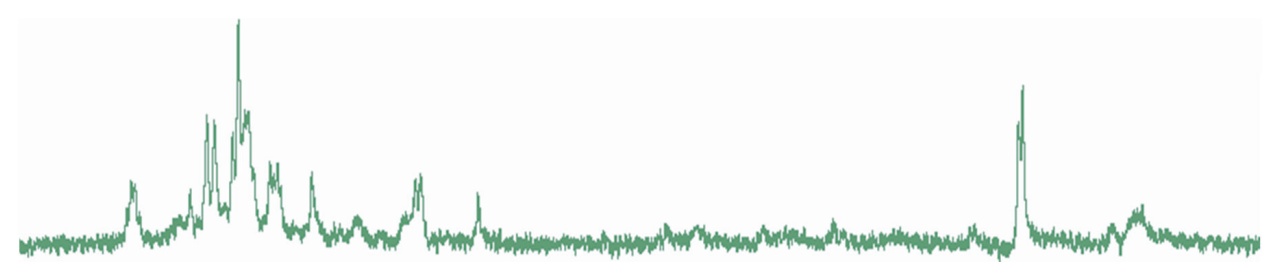

(d)

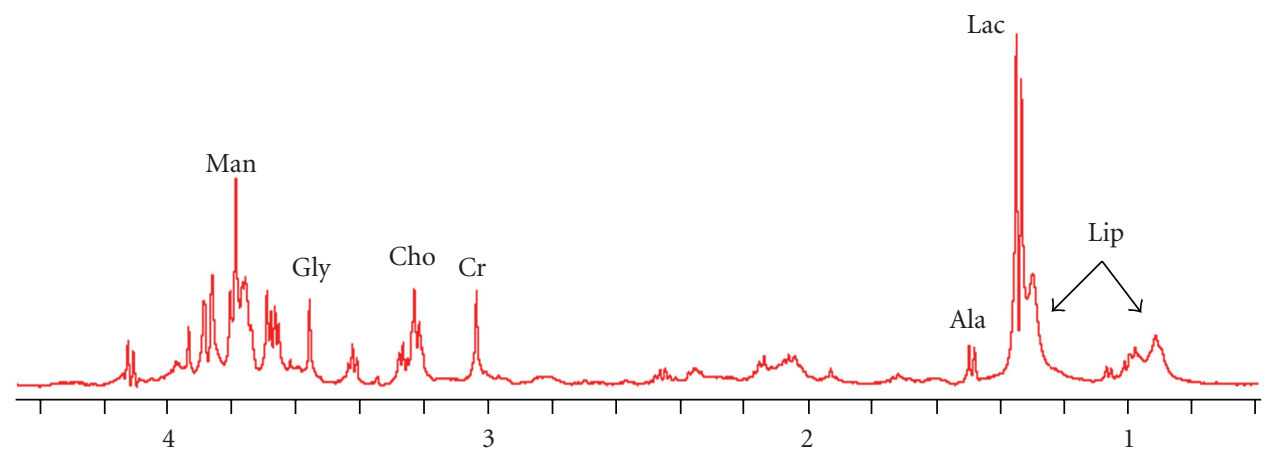

(e)

FIgURE 3: Three orthogonal planes allowed the location of single-voxel in the tissue part (red box) and in the solution (yellow box) for sample G. (a) MRM spectroscopy single-voxel spectra in liquid part, (b) MRM spectroscopy single-voxel spectra in tissue part, (c) the linear combination of spectra (a) and (b), (d) shows the HRMAS CPMG only on the solution collected after the MRM study, and (e) shows the HRMAS CPMG on the whole sample before the MRM study. 
[14], the freezing-thawing and the ischemia processes are not the only ones that can promote changes in the spectra but also the high spinning speed of HRMAS study even at low temperatures. In this type of samples, as $\mathrm{G}$ and $\mathrm{H}$, the expected effect is still higher since the biopsies underwent both freezing-thawing and HRMAS effects, as usually in the metabolomic studies involving HRMAS of biological tissues.

It is also remarkable the different peak width of these resonances coming from the tissue and the solution parts (Figure 2(e), bottom and middle, resp.), with two different contributions to the HRMAS overall spectrum. The effect is a broadening in the base of the peaks (Figure 2(e), top spectrum) that can be a drawback when automated analyses strategies are used. Like the linewidths, also the chemical shifts of metabolites within the tissue and outside in the solution may be different, and this may reduce the accuracy of quantification [16].

\section{Conclusion}

In conclusion, the MRM approach here presented allowed to visualize the great distortion of the brain tumor tissue produced by the HRMAS spinning and to detect a selective transference of metabolites from the tissue to the medium, even in nonstressing osmotic conditions. Our analysis implies that in some cases HRMAS spectra could correspond mainly to a solution and not so to an "intact" tissue. This effect must be accounted for in the subsequent quantification and interpretation of the results of HRMAS spectra in tissues, since the transference from the tissue to the solution is not equivalent for all the metabolites and for all the samples, and can also be influenced by the way in which the sample has been obtained and preserved. It might be expected a different contribution to the HRMAS spectrum of those metabolites transferred to the solution and those which preferentialy remain in the tissue (in fact, the relaxation conditions are characteristically different in the tissue and in the solution, and therefore the shape of the peaks can experiment considerable variations). The MRM analysis provides a connection between the microscopic image and the metabolic composition, yielding spatially localized molecular information that can be relevant in the correlation with other molecular imaging techniques and in the understanding of their biochemical significance.

\section{Acknowledgments}

The authors are grateful for the financial support of Spanish Government SAF2007-6547 and VI Framework E.U. FP62002-LSH 503094' projects.

\section{References}

[1] M. Castillo and L. Kwock, "Clinical applications of proton magnetic resonance spectroscopy in the evaluation of common intracranial tumors," Topics in Magnetic Resonance Imaging, vol. 10, no. 2, pp. 104-113, 1999.
[2] I. M. Burtscher and S. Holtås, "Proton magnetic resonance spectroscopy in brain tumours: clinical applications," Neuroradiology, vol. 43, no. 5, pp. 345-352, 2001.

[3] C. Majós, M. Julià-Sapé, J. Alonso et al., "Brain tumor classification by proton MR spectroscopy: comparison of diagnostic accuracy at short and long TE," American Journal of Neuroradiology, vol. 25, no. 10, pp. 1696-1704, 2004.

[4] A. Stadlbauer, S. Gruber, C. Nimsky et al., "Preoperative grading of gliomas by using metabolite quantification with high-spatial-resolution proton MR spectroscopic imaging," Radiology, vol. 238, no. 3, pp. 958-969, 2006.

[5] B. Celda, D. Monleón, M. C. Martínez-Bisbal et al., "MRS as endogenous molecular imaging for brain and prostate tumors: FP6 project "eTUMOR"', Advances in Experimental Medicine and Biology, vol. 587, pp. 285-302, 2006.

[6] J. Peeling and G. Sutherland, "High-resolution 1H NMR spectroscopy studies of extracts of human cerebral neoplasms," Magnetic Resonance in Medicine, vol. 24, no. 1, pp. 123-136, 1992.

[7] R. J. Maxwell, I. Martínez-Pérez, S. Cerdán et al., "Pattern recognition analysis of $1 \mathrm{H}$ NMR spectra from perchloric acid extracts of human brain tumor biopsies," Magnetic Resonance in Medicine, vol. 39, no. 6, pp. 869-877, 1998.

[8] L. L. Cheng, M. J. Ma, L. Becerra et al., "Quantitative neuropathology by high resolution magic angle spinning proton magnetic resonance spectroscopy," Proceedings of the National Academy of Sciences of the United States of America, vol. 94, no. 12, pp. 6408-6413, 1997.

[9] M. C. Martínez-Bisbal, L. Martí-Bonmatí, J. Piquer et al., “1H and 13C HR-MAS spectroscopy of intact biopsy samples ex vivo and in vivo $1 \mathrm{H}$ MRS study of human high grade gliomas," NMR in Biomedicine, vol. 17, no. 4, pp. 191-205, 2004.

[10] K. S. Opstad, B. A. Bell, J. R. Griffiths, and F. A. Howe, "Toward accurate quantification of metabolites, lipids, and macromolecules in HRMAS spectra of human brain tumor biopsies using LCModel," Magnetic Resonance in Medicine, vol. 60, no. 5, pp. 1237-1242, 2008.

[11] T. E. Sjøbakk, R. Johansen, T. F. Bathen et al., "Characterization of brain metastases using high-resolution magic angle spinning MRS," NMR in Biomedicine, vol. 21, no. 2, pp. 175185, 2008.

[12] M. C. Martínez-Bisbal, D. Monleon, O. Assemat et al., "Determination of metabolite concentrations in human brain tumour biopsy samples using HR-MAS and ERETIC measurements," NMR in Biomedicine, vol. 22, no. 2, pp. 199-206, 2009.

[13] M. Wilson, N. P. Davies, M.-A. Brundler, C. McConville, R. G. Grundy, and A. C. Peet, "High resolution magic angle spinning $1 \mathrm{H}$ NMR of childhood brain and nervous system tumours," Molecular Cancer, vol. 8, article no. 6, 2009.

[14] K. S. Opstad, B. A. Bell, J. R. Griffiths, and F. A. Howe, "An assessment of the effects of sample ischaemia and spinning time on the metabolic profile of brain tumour biopsy specimens as determined by high-resolution magic angle spinning (1)H NMR," NMR in Biomedicine, vol. 21, no. 10, pp. 11381147, 2008.

[15] D. A. Middleton, D. P. Bradley, S. C. Connor, P. G. Mullins, and D. G. Reid, "The effect of sample freezing on proton magicangle spinning NMR spectra of biological tissue," Magnetic Resonance in Medicine, vol. 40, no. 1, pp. 166-169, 1998.

[16] R. Bourne, T. Dzendrowskyj, and C. Mountford, "Leakage of metabolites from tissue biopsies can result in large errors in quantitation by MRS," NMR in Biomedicine, vol. 16, no. 2, pp. 96-101, 2003. 
[17] D. Russell and L. J. Rubinstein, Russel and Rubinstein's Pathology of Tumors of the Nervous System, Arnold, London, UK, 1988.

[18] V. A. Levin, S. A. Leibel, and P. H. Gutin, Cancer Principles and Practice of Oncology, eddited by De Vita V. T. Jr., Devita Hellman S., Rosenberg S. A., Lippincott-Raven, Philadelphia, Pa, USA, 5th edition, 1997. 

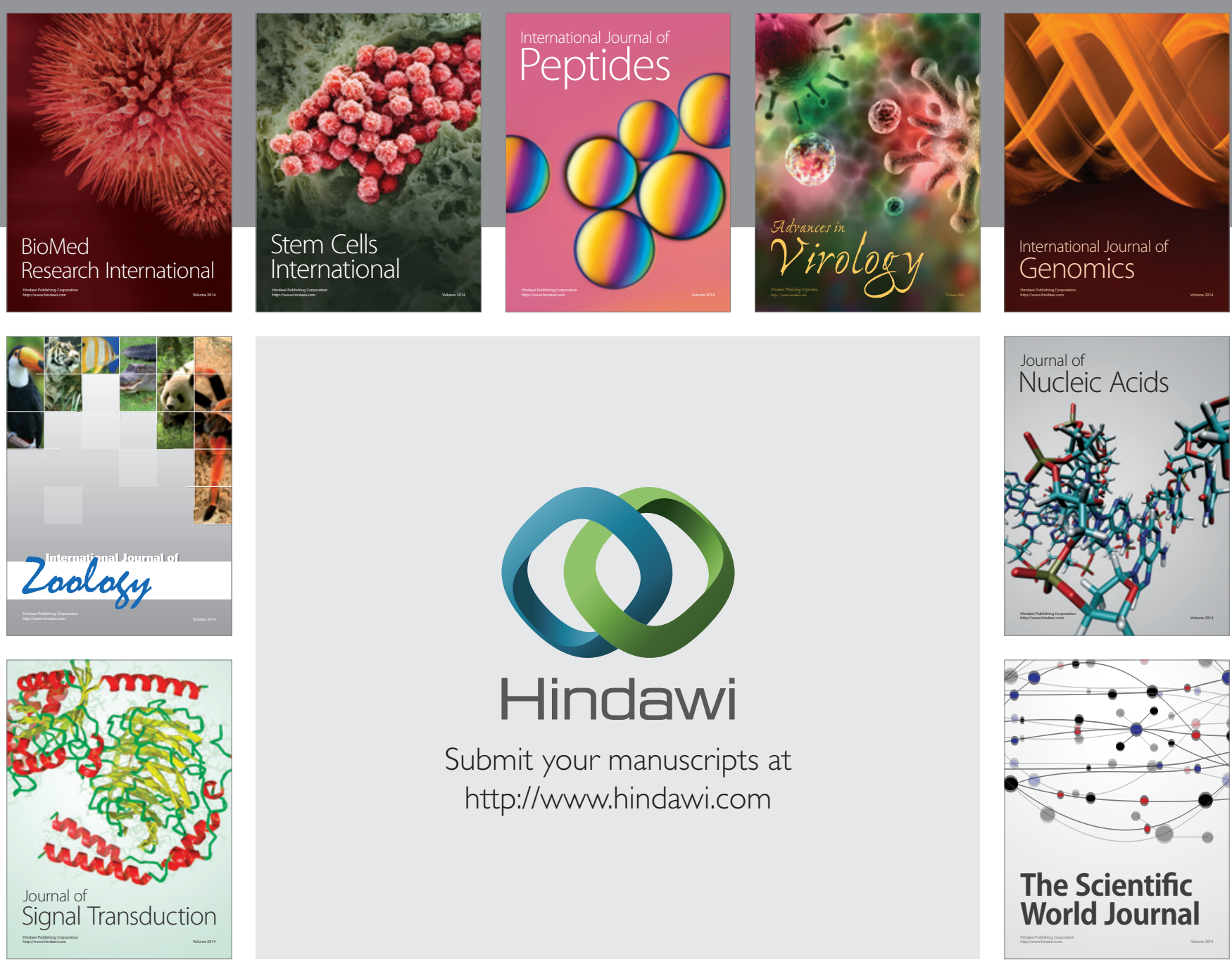

Submit your manuscripts at

http://www.hindawi.com
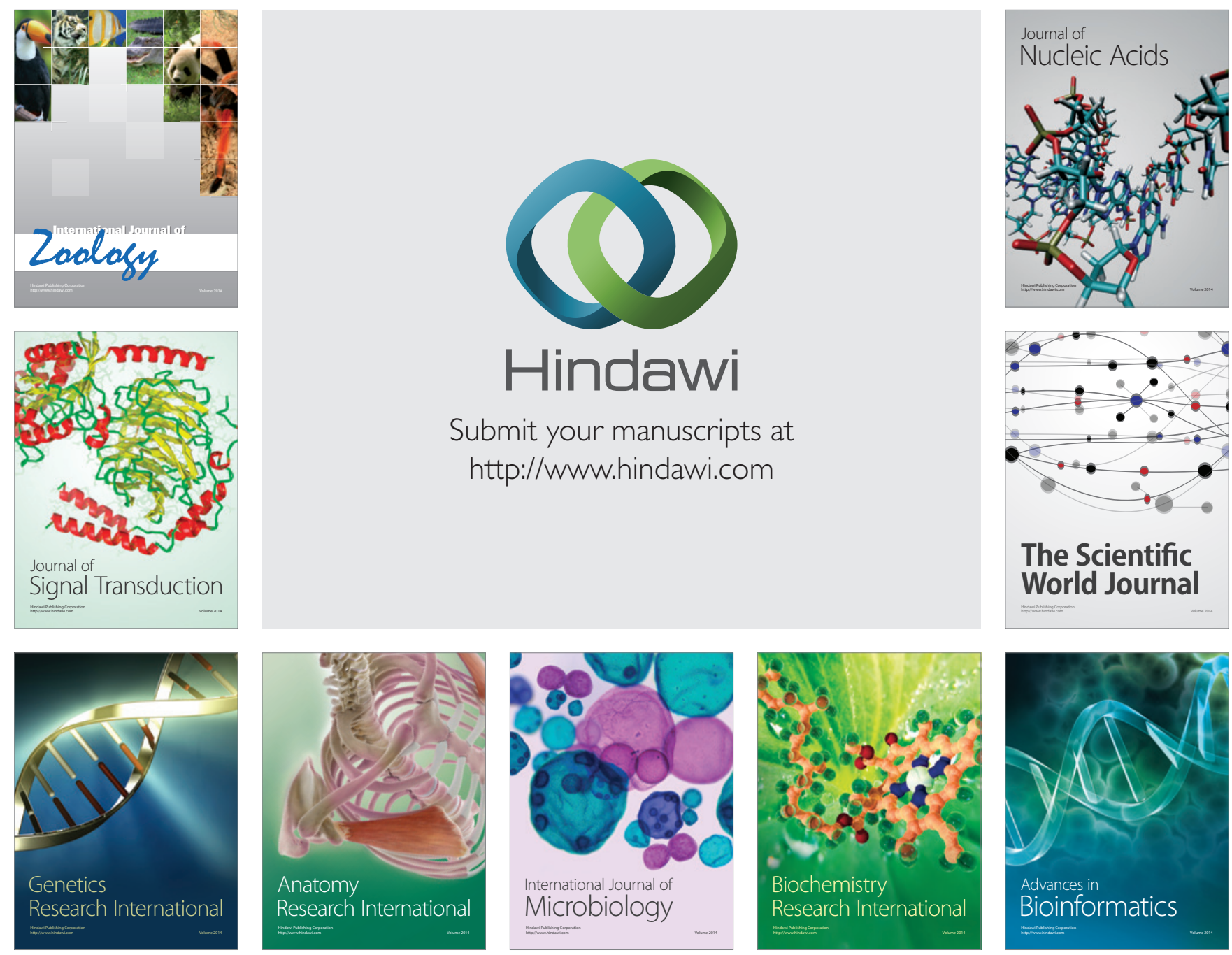

The Scientific World Journal
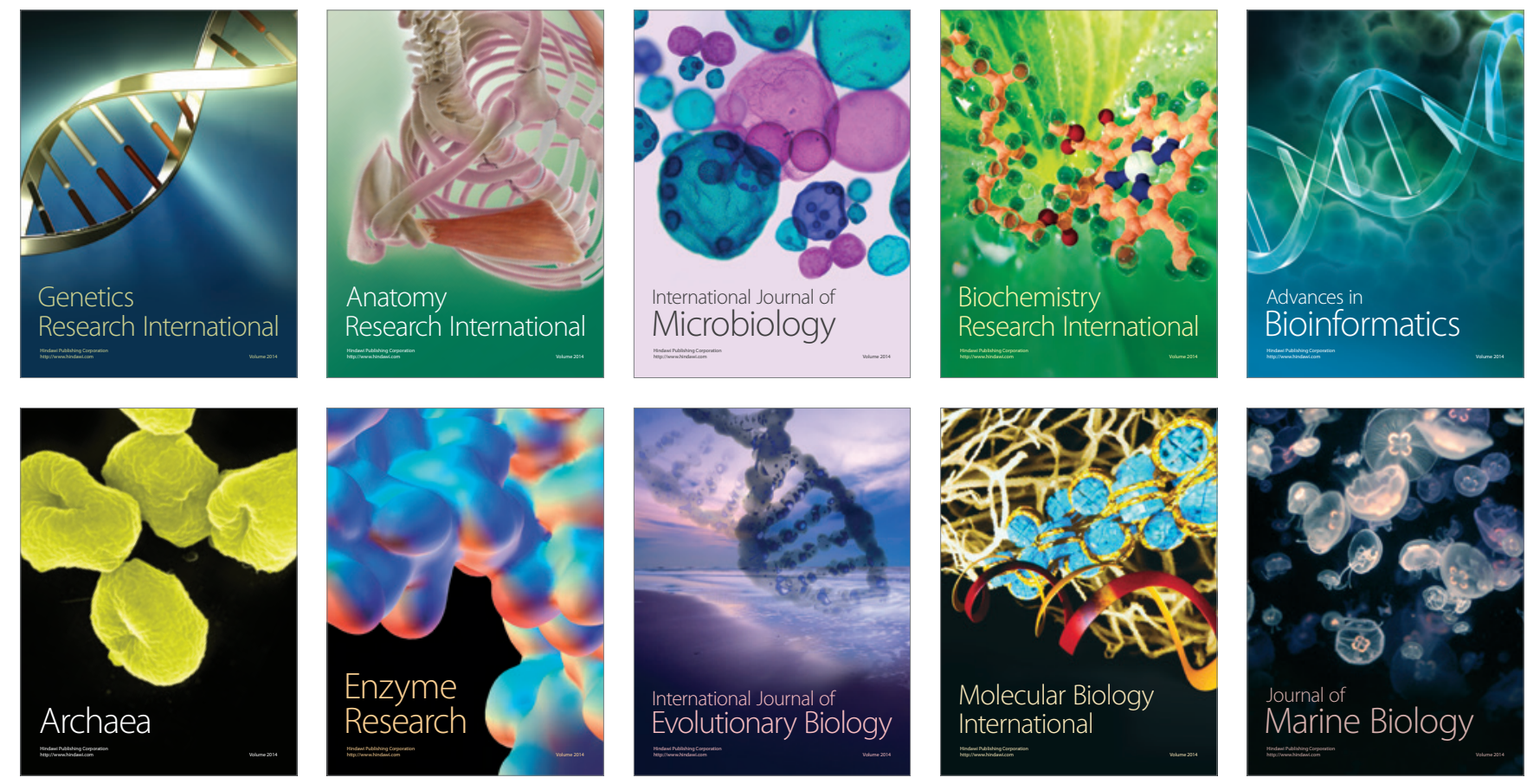